\title{
THE PENN STATE INTELLIGENT DISTRIBUTED CONTROL RESEARCH LABORATORY
}

R.M. Edwards, J.A. Turso

Department of Nuclear Engineering
K.Y. Lee, Senior Member, H.E. Garcia

Department of Electrical and Computer Engineering

The Pennsylvania State University University Park, Pa. 16802
Asok Ray, Senior Member

Department of Mechanical Engineering
Abstract - This paper describes the intelligent distributed controls research laboratory (IDCRL) in the College of Engineering at the Pennsylvania State University (PSU). The center-piece of equipment is a modern distributed microprocessor-based control system which is interfaced to real-time simulations of power plant processes. Research into implementation issues of hierarchical and distributed control for large-scale power plant systems are now more fully explored at the university level. The microprocessor-based control system has also been interfaced to the PSU TRIGA nuclear research reactor and enables research in optimal, robust, intelligent, and other advanced control techniques for nuclear power plants.

Keywords - simulation, microprocessor-based control, intelligent control, robust control, distributed control, hierarchical control, nuclear power plant, TCP/P.

\section{INTRODUCTION}

The distributed microprocessor-based control system is typical of the technology currently being employed to upgrade existing power plant controls and in new construction planning [1]. In most of these real-world situations, incorporation of digital computer-based control is being pursued primarily as a simple change-out of output feedback analog controllers and justified mainly due to the lower cost associated with the improved reliability and maintainability of digital equipment [2]. The Intelligent Distributed Control Research Laboratory (IDCRL) enables research into advanced diagnostic and control concepts for power plant digital control environments. The goal is to derive additional cost benefits associated with improved performance, fault-accommodating control and operational safety that these theoretically-based advanced concepts can potentially provide.

The equipment has been interfaced to simulations of power plant processes presently conducted in mainframe computer systems or in 386 based PC computers. The outputs of a simulated process are placed on the distributed microprocessorbased control system local communication network and picked-up as the inputs to control and diagnostic algorithms executing in the microprocessor-based control system. Advanced diagnostic and control concepts are programmed in the distributed controllers using the $\mathbf{C}$ programming language and their local results are communicated to higher level diagnostic and control functions as well as being used to directly effect control actions in the simulation and ultimately in an actual power plant. The higher

92 WM 075-2 EC A paper recommended and approved by the IEEE Nuclear Power Engineering Committee of the Power Engineering Society for presentation at the IEEE/PES 1992 Winter Meeting, New York, New York, January 26 - 30, 1992. Manuscript submitted September 3. 1991; made available for printing December 10, 1991. level functions can be executed in a hierarchical manner using a variety of computers: mainframes, workstations, or PC computers.

This equipment has also been adapted for creation of a realworld experimental test-bed facility using the Penn State TRIGA research reactor. A TRIGA reactor has the essential non-linear dynamics and stochastic characteristics of a reactor in a commercial pressurized water reactor power plant. This test-bed, which may be expanded to simulate other nuclear power plant characteristics such as boiling heat transfer and multivariable processes, provides an additional focal point for experimental research to better meet real-world implementation concerns where stochastic processes, disturbances, and uncertainties must be simultaneously accommodated in power plant and power system control strategies.

\section{OVERALL SYSTEM}

Figure 1 summarizes the overall structure of the IDCRL which incorporates the distributed microprocessor-based control system. To approach real-time performance on large-scale power plant systems, distributed simulation is utilized [3]. Loosely coupled power plant subsystems such as condensate, feedwater, boiler, and turbine-generator can be individually simulated in separate computers. Coordination of the subsystem simulations is accomplished in a centralized manner in a coordinating computer which maintains a centralized plant data base. Bidirectional communication between the coordinating computer and distributed simulations utilizes TCP/IP on an ETHERNET network. Any simulation computer which can utilize these standard communication features can be incorporated in the IDCRL. Thus far, VAX mainframe computers have been utilized for the distributed simulation needs of our initial research.

The coordinating computer can also, in principle, be any computer system which accommodates TCP/IP communication; however, it has been initially implemented in a UNLX workstation to take advantage of UNIX shared memory, multi-processing, and graphics presentation capabilities. Research into effective utilization of CRT displays for operation and diagnosis of power plant performance is also being conducted $[4,5]$. The local control network indicated in Figure 1 represents the distributed microprocessor-based control system which incorporates a specialized communication system and protocol for local data transfers between microprocessor-based controllers. The GATEWAY computer system is programmed as needed to transfer real-time data between the local control network and the remainder of the IDCRL.

\section{DISTRIBUTED MICROPROCESSOR-BASED CONTROLLERS}

The distributed control system is a Bailey NETWORK 90 system configured into 2 major process control units (PCUs) each individually contained in a separate rack of equipment as summarized in the block diagram in Figure 2. The local 


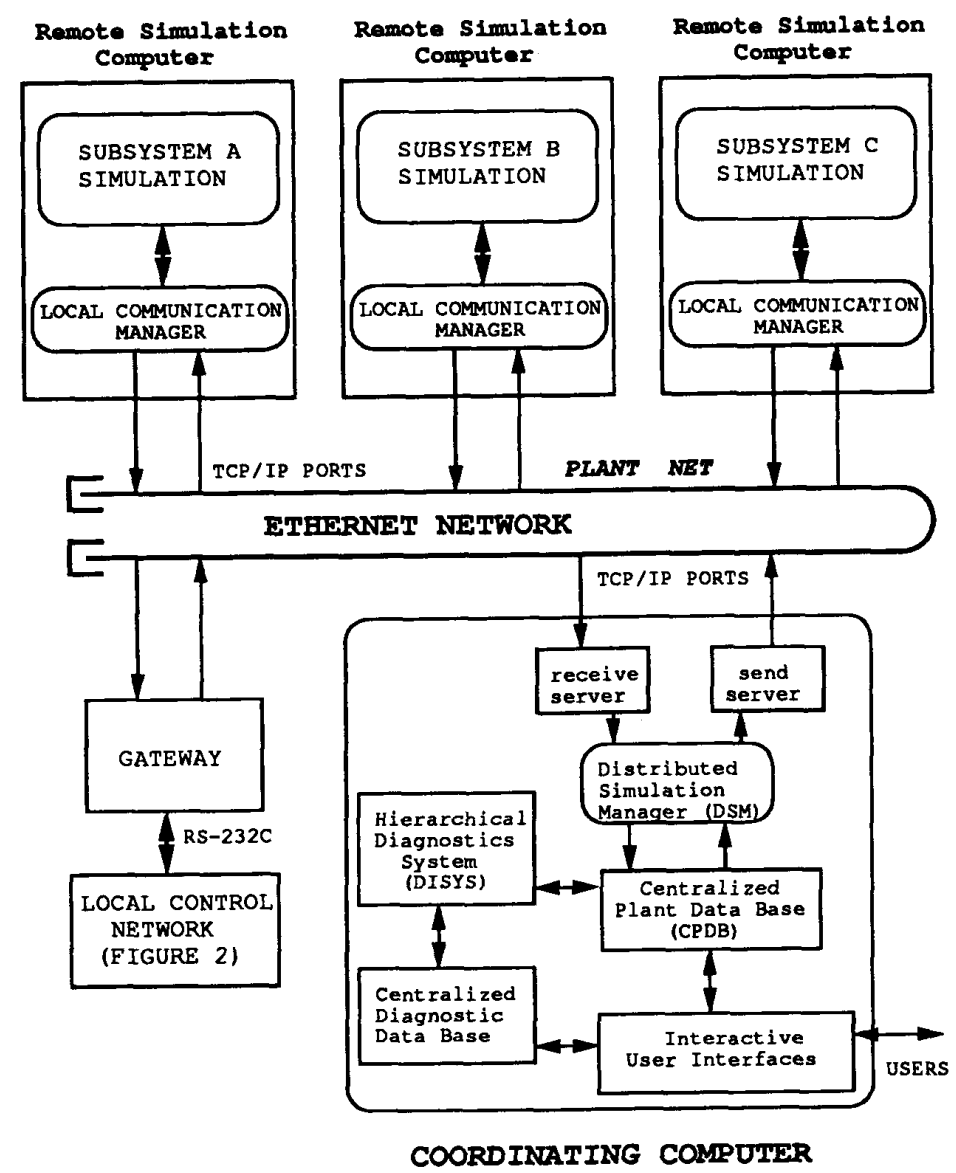

Fig. 1. Configuration of the Penn State Intelligent Distributed Control Laboratory

communication network is a single wire cable (typically implemented redundantly) which provides direct data transfers between PCUs using a Bailey protocol based on exception reporting. A 386 based PC Engineering Workstation (EWS) provides controller programming and monitoring facilities.

Computers external to the local control network, such as the EWS and the IDCRL distributed simulation system, are connected via specialized computer interface unit modules. This connection can be through a serial or IEEE 488 interface. Several microprocessor-based controllers may be contained in a process control unit (PCU) and high speed communication between controllers in the same PCU may also be conducted through a special module data BUS.

Modularized microprocessor-based controller units are classified as standard or multi-function. The standard controller module is programmed using a library of preprogrammed routines identified as control blocks. The control engineer programs the standard controller by selecting the blocks, interconnecting the predefined inputs and outputs, and specifying parameters associated with the control block's function. Multi-function controllers provide the similar control block programming capability and have an expanded capacity for the number of blocks which can be utilized in an active control strategy typically involving a number of control loops which could require dozens of standard controllers. A standard controller has a field Input/Output $(\mathrm{I} / \mathrm{O})$ capability of 4 analog inputs, 2 analog outputs, 3 digital inputs, and 4 digital outputs. Field $1 / O$ for a multifunction controller is provided with up to 64 slave controllers. Each slave has the same field I/O capability as a standard controller. In addition to expanded control block function capability, a multi-function controller provides complete programming capability using the Basic or $\mathrm{C}$ computer language. The $C$ language is utilized in the IDCRL. Typical advanced control applications use a combination of the programming language capability integrated with the standard control block functions [6].

The present IDCRL contains one standard microprocessor-based controller and one multifunction controller and will soon be expanded to two multifunction controllers. 


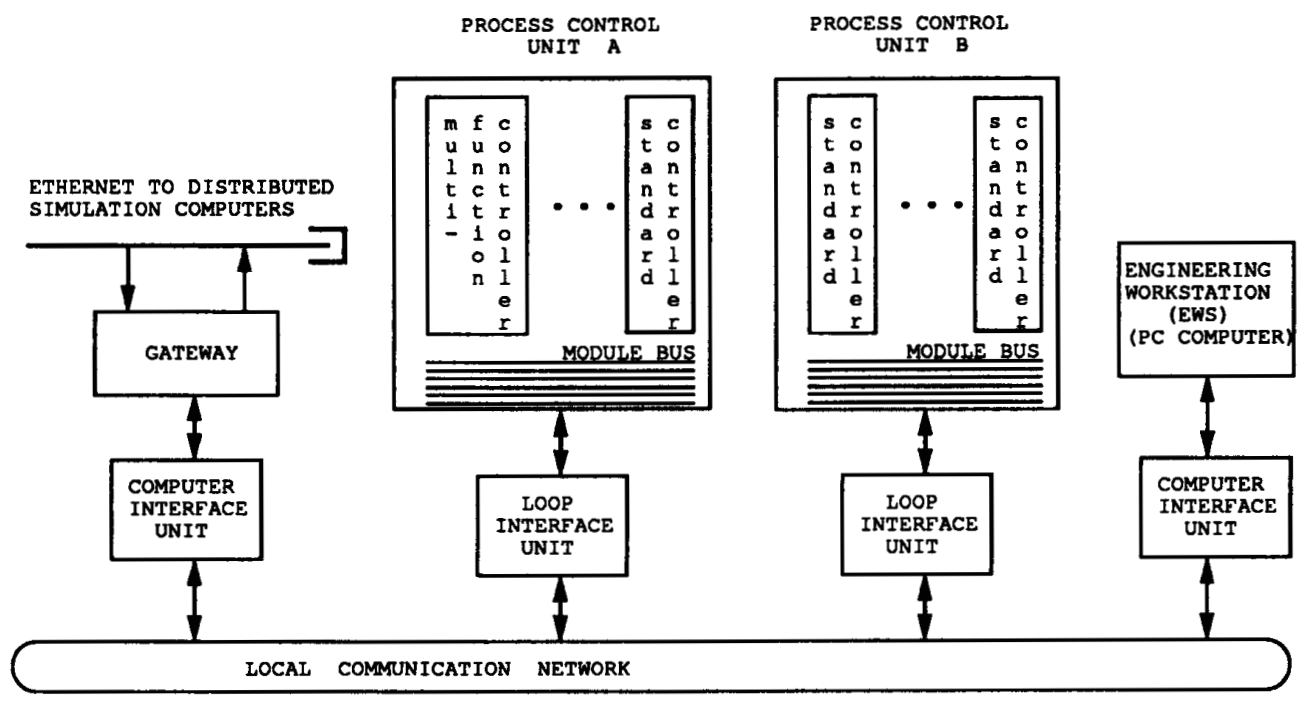

Fig. 2. Local Distributed Control Incorporated in the PSU IDCRL.

\section{INTELLIGENT DISTRIBUTED CONTROL RESEARCH}

The initial research being conducted with the IDCRL is developing an intelligent distributed control demonstration for the Experimental Breeder Reactor (EBR-II) operated by the Argonne National Laboratory at the Idaho National Engineering Laboratory [7]. The steam plant of EBR-II, which produces $20 \mathrm{MWe}$ for the commercial grid, is simulated in the IDCRL as three major subsystems: condensate, feedwater, and steam generation. The condensate and feedwater system has many similarities as central station power plants and has 3 closed feedwater heaters and an open deaerating feedwater heater. The steam generation system is a natural circulation steam drum system where the evaporators and superheaters are heated with liquid sodium. Strategies for accommodating feedwater system faults have been extensively developed and evaluated for demonstration at EBR-II [8-12].

\section{$\underline{\text { Reconfigurable Control }}$}

An intelligent reconfigurable control strategy for accommodating faults in the steam supply to the deaerating heater is demonstrated using all the features of the IDCRL system [10]. Local Proportional-Integral (PI) control algorithms for manipulating the condensate flow into the deaerator are programmed in a multifunction controller. The reconfigurable control strategy uses the $\mathrm{C}$ language programming capability of the controller and chooses between the standard control law for level regulation and an alternate control law for pressure regulation.

Valve position commands determined by the multi-function controller executing the reconfigurable control law are communicated to the condensate system simulation in a VAX computer through the gateway indicated in Figure 1. The simulated response of the deaerator pressure and level process variables are communicated to the centralized plant data base of the coordinating computer and to the multifunction controller which is executing the intelligent reconfigurable control strategy.
By monitoring and evaluating the performance of the deaerator pressure and level process variables, the reconfigurable control strategy uses a learning systems approach to choose the control law for the condensate flow control valve. The concept accommodates a loss of steam supply fault by switching to the alternate pressure control for manipulation of the condensate valve. Its effectiveness has been demonstrated by simulation with the IDCRL and planning for an actual experiment at EBR-II is underway.

\section{Robust Control}

A multivariable robust controller design has also been examined for accommodating feedwater system faults $[11,12]$. By designing the robust controller in the State Feedback Assisted Classical Control Configuration [13], the robust controller accommodates a deaerator loss of steam supply fault by simply lowering the level setpoint to an embedded level control law for manipulating the condensate valve position. Utilization of an embedded conventional controller within a state feedback system has also been shown to offer improved robustness characteristics over implementation of a state feedback system without the embedded conventional feedback controller [14]

The intelligent reconfigurable controller performance can be improved by incorporating the possibility of selecting multivariable robust controllers in addition to the conventional single loop PI control laws.

\section{Hierarchical Distributed Intelligent Control}

Current research examines implementation of reconfigurable, robust, and intelligent control for the remainder of the EBR-II steam plant, in particular, steam drum level and steam header pressure control. Localized intelligent control for individual subsystems will be coordinated in a heirachical manner in a structured manner very similar to the coordination of the existing IDCRL distributed simulation system. 
This work will necessarily include an assessment of the effects of the time response of the data highways and the interconnecting gateway on the simulation time and accuracy. At the lowest levels of a heirachical distributed control system localized advanced controllers, such as the multivariable robust controller, will be executed in dedicated microprocessor-based controllers where the time response of the network does not impact the performance of the controller. Increasingly at higher levels in the hierarchy, intelligent control actions may be impacted by time delays caused by the network and must be taken into account in order to insure stability and performance of the overall system.

\section{TRIGA REACTOR TEST-BED FACILITY}

The Penn State TRIGA research reactor is a light water cooled and reflected pool type reactor capable of both pulsing to $2000 \mathrm{MW}$ and constant power operation at $1 \mathrm{MW}$ maximum. The technical specifications for operation of the TRIGA reactor have been carefully considered in the process of specifying and installing a new digital control and monitoring system [15]. An experimental procedure for testing control algorithms and hardware uses a secondary control rod and instrumentation system while supervisory and safety functions are provided by the licensed control and monitoring system. The secondary control rod's capability to change reactor power (magnitude and rate) are selected based on the constraints of the reactor technical specifications; however, due to the inherent ultra-safe characteristics of the TRIGA research reactor, these constraints still provide more than enough flexibility to conduct meaningful advanced digital control experiments.

The dynamics of the TRIGA reactor are represented by the point kinetics equations and a single temperature reactivity feedback mechanism [16]. In control experiments, the reactor power is measured with a compensated ionization chamber and digitized for use in a microprocessor-based control system. The output of the controller is a speed demand signal for the secondary control rod. Initial experiments have been conducted to demonstrate an optimal control algorithm to improve temperature response of the TRIGA reactor [17]. This optimal control algorithm has also been studied for application to pressurized water reactors [18] and liquid metal reactor power plants with simulation. The TRIGA reactor experiments are thus the first experimental verification of the optimal temperature control algorithm's validity. Reactor applications of fuzzy control and neural network control [19] are additionally being examined for demonstration and testing on the TRIGA reactor.

A hybrid test-bed facility which combines simulation with the PSU TRIGA reactor is also under consideration [15]. Physical reactivity mechanisms which are not characteristic of the TRIGA reactor, such as boiling heat transfer or a positive temperature coefficient, will be simulated and used to adjust a secondary control rod in the TRIGA reactor. Control algorithms to improve performance of reactor power plant systems with the simulated characteristics can then be tested with the hybrid facility. The proposed hybrid TRIGA/simulation testing facility is analogous to the North Carolina State University Freon Pressurized Water Reactor test-loop [20]. In that facility, the reactor kinetics portion of a power plant is simulated with a digital computer and a physical model is used to obtain the thermal-hydraulic response of the power plant. In the TRIGA/simulation facility, the complementary operation is performed where the thermal/hydraulics is simulated while a physical system generates the reactor kinetics response.

\section{CONCLUSIONS}

The Penn State Intelligent Distributed Control Laboratory is providing new opportunities for university research into advanced control, diagnosis, and operation of power plants (nuclear and fossil). The system, which incorporates distributed simulation and a distributed microprocessor-based control system, is being applied to the development of intelligent distributed control for demonstration at the Experimental Breeder Reactor. Initial experiments using the Penn State TRIGA reactor are also leading the way to the development of a hybrid testing facility which combines simulated processes with the actual dynamic response of the research reactor.

\section{ACKNOWLEDGEMENTS}

The development of a demonstration of Intelligent Distributed Control for the EBR-II steam plant is being conducted as part of a DOE university program grant, DEFG-07-89ER 12889. The Penn State Bailey NETWORK 90 system which established the IDCRL was acquired as an NSF grant, ECS-8905917. However, any findings, conclusions, or recommendations expressed herein are those of the authors and do not necessarily reflect the views of DOE or NSF.

\section{REFERENCES}

[1] E.S. Kenney, R.M. Edwards, K.Y. Lee, Asok Ray, and S.T. Kumara, FINAL Technical Report: Engineering Research Equipment Grant - Microprocessor-based Controllers, NSF Grant ECS-8905917, January 1991.

[2] S. Bhatt, J. Naser, L. Oakes, W. Reuland, J. Weiss, and D. Wilkinson, "Bridging the I\&C Gap for Nuclear Plants," EPRI Journal, December 1990, pp 36-39.

[3] R.M. Edwards, J.A. Turso, H.E. Garcia, S. Dharap, and S. Lee, "Real-time Distributed Simulation Using the Modular Modeling System Interfaced to a Bailey NETWORK 90 System," EPRI 1991 International Conference on Power Plant \& Power System Training, Simulators, \& Modeling, Miami Beach, Florida, April 1991.

[4] R.M. Edwards, C. Chavez, S. Kamarthi, S. Dharap, R.W. Lindsay, and J. Staffon, "The Graphics-Based Human Interface to the DISYS Diagnostic and Control Guidance Expert System at EBR-II," Proceedings of the 1990 International Fast Reactor Safety Meeting, Snowbird, Utah, Vol. III, pp 309-316, August 1990.

[5] C.M. Chavez, J.H. Goldberg, and R.M. Edwards,"A Human Factors Evaluation of a Highly Automated Nuclear Power Plant," AI 91: Frontiers in Innovative Computing for the Nuclear Industry, Jackson, Wyoming, September 1991.

[6] Gerald L. Stephens, "Advanced Process Control with INFI 90," Control Engineering, Vol. 35, No. 9, September 1988.

[7] R.M. Edwards, D.W. Ruhl, E.H. Klevans, and G.E. Robinson, "Development and Testing of a Diagnostic System for Intelligent Distributed Control at EBR-II," Proceedings of the 1990 International Fast Reactor Safety Meeting, Snowbird, Utah, Vol. III, pp 299-308, August 1990.

[8] H.E. Garcia, Asok Ray, and R.M. Edwards, "A Reconfigurable Control Strategy for Distributed Digital Process Control," Proceedings of the Sixth Yale Workshop on Adaptive and Learning Systems, pp 184-188, Yale University, Connecticut, August 1990.

[9] H.E. Garcia, Asok Ray, and R.M. Edwards, "Reconfigurable Control of Power Plants Using Learning Automata," IEEE Control System Magazine, Vol. 11, No. 1, pp 85-92 (1991). 
[10] H.E. Garcia, R.M. Edwards, Asok Ray, and E.H. Klevans, "On-Line Diagnostics and Intelligent Control for the Steam Plant at EBR-II," Al 91: Frontiers in Innovative Computing for the Nuclear Industry, Jackson, Wyoming, September 1991.

[11] R.M.Edwards, A. Ben-Abdennour, and K.Y. Lee, "Robust Control Design Verification Using the Modular Modeling System," EPRI 1991 International Conference an Power Plant \& Power System Training, Simulators, \& Modeling, Miami Beach, Florida, April 1991.

[12] A. Ben-Abdennour, R.M. Edwards, and K.Y. Lee,"MIMOML Robust Control for Accommodating EBR-II Feedwater System Faults," AI 91: Frontiers in Innovative Computing for the Nuclear Industry, Jackson, Wyoming, September 1991.

[13] R.M. Edwards, K.Y. Lee, and M.A. Schultz, "State Feedback Assisted Classical Control: An Incremental Approach to Control Modernization of Existing and Future Nuclear Reactors and Power Plants," Nuclear Technology, Vol. 92, pp 167-185, November 1990.

[14] R.M. Edwards, K.Y. Lee, and Asok Ray, "Multi-Layer Robust Control for Safety Enhancement of Reactor Operations," Transactions of the American Nuclear Society, Vol. 63, pp 402-403, June 1991.

[15] Dan Hughes, Mac Bryan, and Robert Gould, "Safety Issues Involved in Using the PSBR for Control Algorithm Testing," AI 91: Frontiers in Innovative Computing for the Nuclear Industry, Jackson, Wyoming, September 1991.

[16] M.A. Schultz, Control of Nuclear Reactors and Power Plants, 2nd ed., McGraw-Hill Book Company, New York (1961).

[17] J.A. Turso, R.M. Edwards, Dan Hughes, Mac Bryan, and H.E. Garcia, "Experience With Developing a Real World Advanced Control and Diagnostic Testbed Using a University Research Reactor," AI 91: Frontiers in Innovative Computing for the Nuclear Industry, Jackson, Wyoming, September 1991.

[18] R.M. Edwards, "Robust Optimal Control of Nuclear Reactors," Ph.D. Dissertation in Nuclear Engineering, The Pennsylvania State University, June 1991.

[19] C.C. Ku, K.Y. Lee, and R.M. Edwards, "A Neural Network for Adapting Nuclear Power Plant Operation for Wide Range Operation," Transactions of the American Nuclear Society, Vol. 63, pp 114-115, June 1991.

[20] J.R. Caves, G.D. Miller, and B.W. Wehring, "NCSU Pressurized Water Reactor Physical Simulator," IEEE Transactions on Nuclear Science, vol. 36, pp 1690-1695, October 1990.

\section{BIOGRAPHY}

Robert M. Edwards was born in DuBois, Pennsylvania, January 15,1950 . He received the B.S. degree in Nuclear Engineering from the Pennsylvania State University, University Park, in 1971, the M.S. degree in Nuclear Engineering from the University of Wisconsin, in 1972, and the Ph.D. degree in Nuclear Engineering from the Pennsylvania State University, in 1991.

He has recently become an Assistant Professor of Nuclear Engineering at Penn State, following 4 years as a full-time research assistant. Prior to returning to Penn State, he was the director of software development at LeMont Scientific, State College, $\mathrm{Pa}$. In the early $1970 \mathrm{~s}$, he was employed at General Atomics, San Diego. His research interests are in control and artificial intelligence applications for power plants.

Dr. Edwards has been a member of the American Nuclear Society and the Society for Computer Simulation. He is also a registered Professional Engineer.
James A. Turso received the B.S. degree in Marine Engineering from the State University of New York Maritime College, Fort Schuyler, Bronx, New York, in 1984, the M.S. degree in Mechanical Engineering from Manhattan College, Bronx, New York, in 1990, and is currently pursuing a Doctoral degree in Nuclear Engineering at the Pennsylvania State University.

His several years of industrial experience include testing of shipboard engineering systems as well as shipboard propulsion plant operation. Prior to continuing his education, he was employed as process simulator design engineer.

Mr. Turso is also a New York State Engineer in Training and holds a United States Coast Guard Third Assistant Engineer's license.

Kwang Y. Lee was born in Pusan, Korea, on March 6, 1942. He received the B.S. degree in Electrical Engineering from Seoul National University, Seoul, Korea, in 1964, the M.S. degree in Electrical Engineering from North Dakota State University, Fargo, in 1968, and the $\mathrm{Ph} . \mathrm{D}$. degree in System Science from Michigan State University, East Lansing, in 1971.

He has been on the faculties of Michigan State University, Oregon State University, University of Houston, and the Pennsylvania State University, where he is an Associate Professor of Electrical Engineering. His areas of interest are system theory and its application to large scale system, and power system.

Dr. Lee has been a member of IEEE Control System Society, Power Engineering Society, and Systems Man and Cybernetics Society. He is also a registered Professional Engineer.

Humberto E. Garcia received the Electrical Engineering Diploma from the Universidad de Carabobo in 1985, the M.S. degree in Electrical Engineering from the Pennsylvania State University, University Park, in 1989, and is a Ph.D. candidate in Electrical Engineering at the Pennsylvania State University. His current research interests include distributed intelligent systems, intelligent supervisory and control systems, decision support systems, discrete event dynamical systems, computerized automation and artificial intelligence.

Mr. Garcia is a member of Tao Beta Pi.

Asok Ray earned a Ph.D. degree in Mechanical Engineering and graduate degrees in each of Electrical Engineering, Computer Science, and Mathematics. He has held research and management positions at GTE Strategic Systems Division, Charles Stark Draper Laboratory, and MITRE Corporation as well as academic positions at Carneige-Mellon University and the Massachusetts Institute of Technology. He joined the Pennsylvania State University in July 1985, and is currently a Professor of Mechanical Engineering. His research experience includes real-time microcomputer-based control and instrumentation, networking, and communication protocols, intelligent systems design, and modeling and simulation of dynamical systems as applied to aeronautics, process control, and autonomous manufacturing. Current research interests include distributed control systems, applied stochastic processes and fault detection, and intelligent instrumentation and computer networking for aeronautical and manufacturing systems.

Dr. Ray has authored or co-authored over one hundred and sixty research publications, and is an Associate Fellow of AIAA, a senior member of IEEE and a member of ASME. 